\title{
ERRATUM
}

\author{
Iwajlo M. Kandjov
}

\section{Thermal stability of the human body under hyperbaric environmental conditions: a theoretical study}

Published online: 18 December 2001

(C) Springer-Verlag 2001

Eur J Appl Physiol (2001) 85:572-577

As a result of technical problems, some of the abbreviations in Table 1 were wrong. A correct version of the table appears below.

Table 1 Definitions of abbreviations used in the text

\begin{tabular}{|c|c|c|}
\hline Abbreviation & Definition & Units \\
\hline ATA & Atmospheres absolute & atm \\
\hline $\mathrm{c}$ & Constant in heat transfer equations & None \\
\hline$C$ & Convective heat loss from body surface & $\mathrm{W} \cdot \mathrm{m}^{-2}$ \\
\hline$d$ & Diameter of man equivalent cylinder & $\mathrm{m}$ \\
\hline $\mathrm{D}$ & Diffusion coefficient of water vapour in gas mixture & $\mathrm{m}^{2} \cdot \mathrm{s}^{-1}$ \\
\hline $\mathrm{D}_{\mathrm{o}}$ & Diffusion coefficient of water vapour in air & $\mathrm{m}^{2} \cdot \mathrm{s}^{-1}$ \\
\hline $\mathrm{e}_{\mathrm{a}}$ & Water vapour pressure in ambient air & $\mathrm{mmHg}$ \\
\hline $\mathrm{e}_{\mathrm{s}}$ & Water vapour pressure on body surface & $\mathrm{mmHg}$ \\
\hline$g$ & Gravitational constant & $\mathrm{m} \cdot \mathrm{s}^{-2}$ \\
\hline $\mathrm{Gr}$ & Grashof number $\left[\left(\beta \cdot g \cdot d^{3} \cdot \Delta \mathrm{T}\right) \cdot(v)^{-2}\right]$ & None \\
\hline$h_{c}$ & Convective heat transfer coefficient in gas mixture & $\mathrm{W} \cdot \mathrm{m}^{-2} \cdot{ }^{\circ} \mathrm{C}^{-1}$ \\
\hline $\mathrm{h}_{\mathrm{co}}$ & Convective heat transfer coefficient in air & $\mathrm{W} \cdot \mathrm{m}^{-2} \cdot{ }^{\circ} \mathrm{C}^{-1}$ \\
\hline$h_{D}$ & Mass transfer coefficient & $\mathrm{m} \cdot \mathrm{s}^{-1}$ \\
\hline$h_{e}$ & Evaporative heat transfer coefficient in gas mixture & $\mathrm{W} \cdot \mathrm{m}^{-2} \cdot \mathrm{mmHg}^{-1}$ \\
\hline $\mathrm{h}_{\mathrm{eo}}$ & Evaporative heat transfer coefficient in air & $\mathrm{W} \cdot \mathrm{m}^{-2} \cdot \mathrm{mmHg}^{-1}$ \\
\hline$I$ & Heat flow of solar radiation on human body surface & $\mathrm{W} \cdot \mathrm{m}^{-2}$ \\
\hline $\mathrm{k}$ & Thermal conductivity of gas mixture & $\mathrm{W} \cdot \mathrm{m}^{-1} \cdot{ }^{\circ} \mathrm{C}^{-1}$ \\
\hline $\mathrm{k}_{\mathrm{o}}$ & Thermal conductivity of atmospheric air & $\mathrm{W} \cdot \mathrm{m}^{-1} \cdot{ }^{\circ} \mathrm{C}^{-1}$ \\
\hline LR & Lewis relationship $\left(\mathrm{h}_{\mathrm{e}} \cdot \mathrm{h}_{\mathrm{c}}^{-1}\right)$ & ${ }^{\circ} \mathrm{C} \cdot \mathrm{mmHg}^{-1}$ \\
\hline M & Metabolic heat production & $\mathrm{W} \cdot \mathrm{m}^{-2}$ \\
\hline $\mathrm{Nu}$ & Nusselt number $\left[\left(\mathrm{h}_{\mathrm{c}} \cdot d\right) \cdot \mathrm{k}^{-1}\right]$ & None \\
\hline$P$ & Ambient pressure in gas mixture environment & ATA \\
\hline$P_{\mathrm{o}}$ & Atmospheric pressure at sea level & 1 ATA \\
\hline $\operatorname{Pr}$ & Prandtl number $\left(v_{\mathrm{n}} \cdot \mathrm{a}^{-1}\right)$ & none \\
\hline $\mathrm{R}$ & Radiative heat loss & $\mathrm{W} \cdot \mathrm{m}^{-2}$ \\
\hline $\operatorname{Re}$ & Reynolds number $\left[(v \cdot d) \cdot v_{\mathrm{n}}^{-1}\right]$ & none \\
\hline RHL & Respiratory heat loss & $\mathrm{W} \cdot \mathrm{m}^{-2}$ \\
\hline Sh & Sherwood number $\left[\left(\mathrm{h}_{\mathrm{D}} \cdot d\right) \cdot \mathrm{D}^{-1}\right]$ & None \\
\hline$\Delta \mathrm{T}$ & Temperature difference at the body-gas interface & ${ }^{\circ} \mathrm{C}$ \\
\hline$U$ & Sum of heat flows & $\mathrm{W} \cdot \mathrm{m}^{-2}$ \\
\hline$\beta$ & Coefficient of volume expansion of air & ${ }^{\circ} \mathrm{C}_{2}^{-1}$ \\
\hline$v$ & Kinematic viscosity of mixture & $\mathrm{m}^{2} \cdot \mathrm{s}^{-1}$ \\
\hline$v_{\mathrm{o}}$ & Kinematic viscosity of atmospheric air & $\mathrm{m}^{2} \cdot \mathrm{s}^{-1}$ \\
\hline
\end{tabular}

The online version of the original article can be found at http://dx. doi.org/10.1007/s004210100412

I.M. Kandjov

Medical Climatology Department, National Centre of Physical

Therapy and Rehabilitation, 2-v Ovcha Kupel Blvd,

1618 Sofia, Bulgaria

E-mail: ncflr@datacom.bg

Tel.: + 359-2-9557640; Fax: + 359-2-553023 